\title{
Erratum to: MRI and CT evaluations of an invented bioglue in experimentally induced articular cartilage defects in rabbits
}

\author{
Nader Tanideh • Mohammad Hadi Bagheri • \\ Seifollah Dehghani Nazhvani • Behrooz Nikahval • \\ Fereidoon Mojtahed Jaberi • Davood Mehrabani
}

Published online: 12 December 2013

(C) Springer-Verlag London 2013

\section{Erratum to: Comp Clin Pathol}

DOI 10.1007/s00580-013-1819-0

The original version of this article in advertently contained a mistake. The corrected figures and the corresponding legends are as in this erratum article.

The online version of the original article can be found at http://dx.doi.org/ 10.1007/s00580-013-1819-0.

N. Tanideh $(\square)$

Stem Cell and Transgenic Technology Research Center and

Department of Pharmacology, Shiraz University of Medical

Sciences, Shiraz, Iran

e-mail: nadertanideh@yahoo.com

M. H. Bagheri

Department of Radiology, Shiraz University of Medical Sciences,

Shiraz, Iran

S. D. Nazhvani • B. Nikahval

Department of Veterinary Surgery, School of Veterinary Medicine,

Shiraz University, Shiraz, Iran

F. M. Jaberi

Stem Cell and Transgenic Technology Research Center and

Department of Orthopedic Surgery, Shiraz University of

Medical Sciences, Shiraz, Iran

D. Mehrabani

Stem Cell and Transgenic Technology Research Center and Gastroenterohepatology Research Center, Shiraz University of

Medical Sciences, Shiraz, Iran 


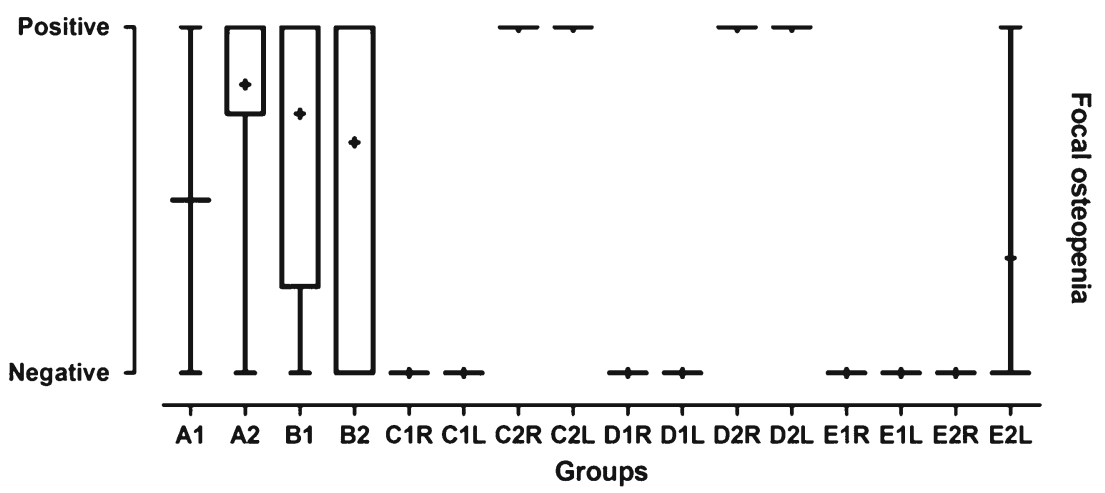

Fig. 1 Box and whisker plot of focal osteopenia detected by CT in different groups. A1 showed significantly higher values when compared with other groups C1R, C1L, D1R, D1L, E1R and E1L. A2 showed significantly lower values when compared with C2L, D2R, D2L, and higher values when compared with E2R and E2L. As compared to B1, groups C1L, D1R, D1L, E1R, E1L, showed significantly lower values.
B2 was significantly lower than groups C2R, C2L, D2R, D2L. E2R and E2L were significantly lower than B2. C1R was lower than C2R. C1L was lower than C2L. E2R was lower than D2R and C2R. E2L was lower than C2L and D2L. D1R was lower than D2R. D1L was lower than D2L. E1L was lower than E2L. E2R was lower than E2L

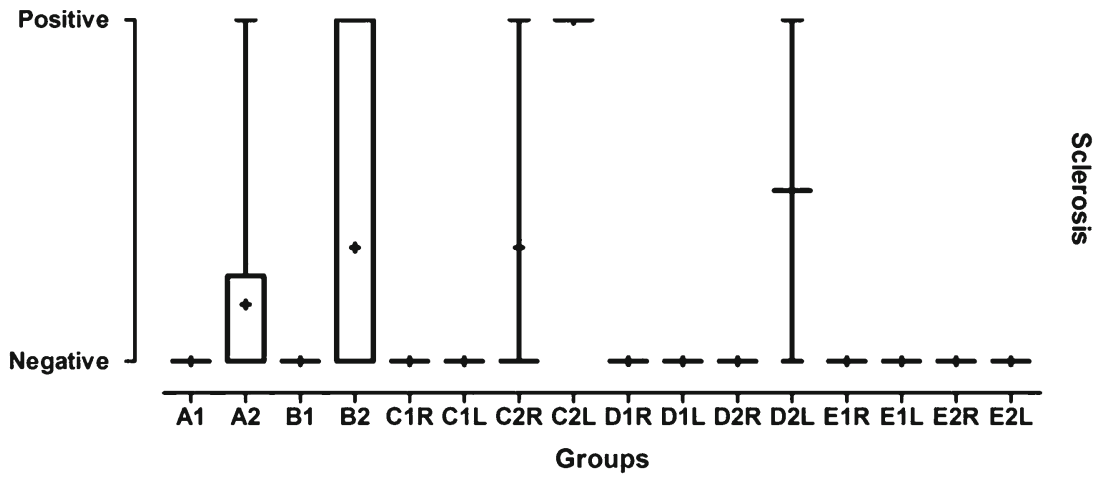

Fig. 2 Box and whisker plot of sclerosis detected by CT in different groups. A2 showed significantly lower values than $\mathrm{C} 2 \mathrm{~L}$ and higher values than E2R and E2L. B1 was lower than B2. B2 was significantly lower than $\mathrm{C} 2 \mathrm{~L}$ and higher than B1, D2R, E2R and E2L. C1R and C1L were lower than $\mathrm{C} 2 \mathrm{R}$ and $\mathrm{C} 2 \mathrm{~L}$ respectively. $\mathrm{C} 2 \mathrm{R}$ was lower than $\mathrm{C} 2 \mathrm{~L}$ and higher than D2R and E2R. C2L was higher than D2L and E2L. D2L was higher than D1L and D2R. E2L was significantly lower than D2L
Fig. 3 Box and whisker plot of spur, subchondral cyst formation and joint space narrowing by $\mathrm{CT}$ in different groups. A2 showed significantly higher values than B2, C2L, D2L and E2L

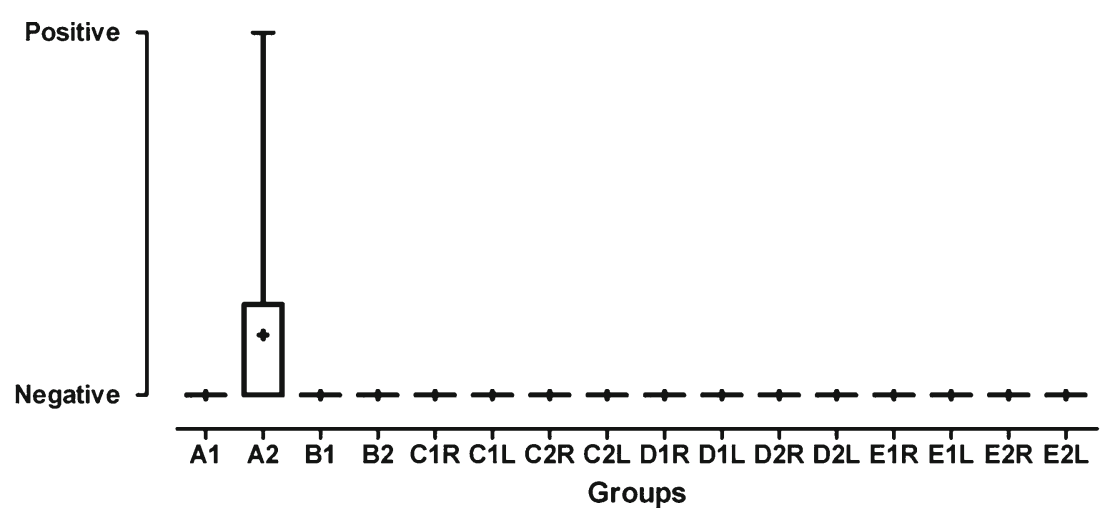




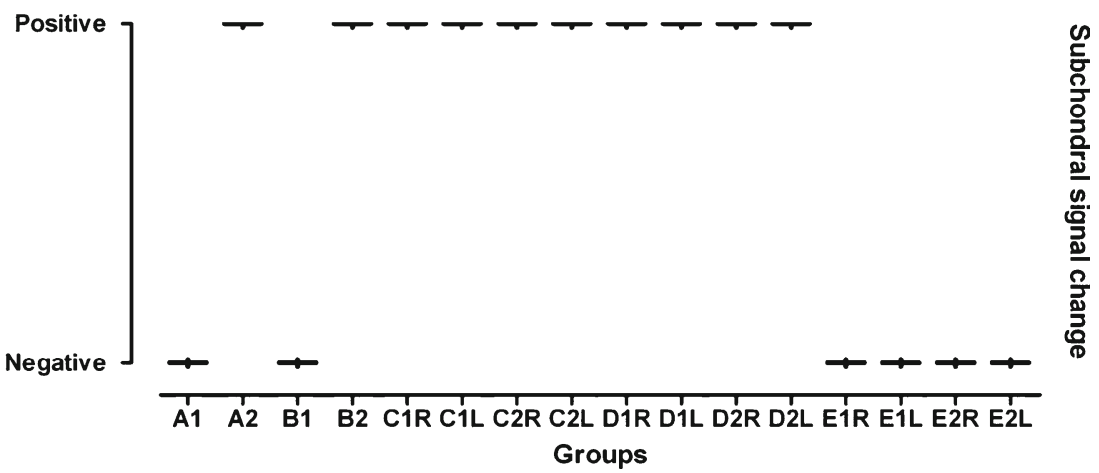

Fig. 4 Box and whisker plot of subchondral signal change detected by MRI in different groups. A1 values were significantly lower than A2, C1R, C1L, D1L, D1R. A2 showed significantly higher values than E2R and E2L. B1 showed significantly lower values than B2, C1R, C1L, D1R and D1L. B2 showed higher values as compared with E2R and E2L. E1R values were significantly lower than C1R and D1R. E1L was significantly lower than C1L and D1L. E2R values were significantly lower than $\mathrm{C} 2 \mathrm{R}$ and $\mathrm{D} 2 \mathrm{R}$. E2L was significantly lower than C2L and D2L

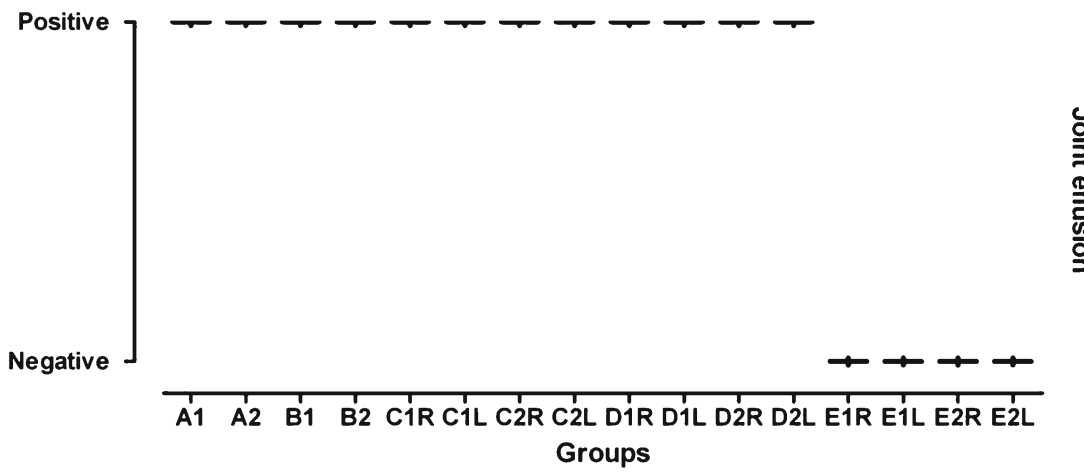

Fig. 5 Box and whisker plot of joint effusion detected by MRI in different groups. A1 showed significantly higher values than E1R, E1L. A2 showed higher values than E2R and E2L. B1 was significantly higher than E1R and E1L. B2 was higher than E2R and E2L. E1R was significantly better than C1R and D1R. E1L was significantly better than $\mathrm{C} 1 \mathrm{~L}$ and D1L. E2R was significantly better than C2R and D2R. E2L was significantly better than $\mathrm{C} 2 \mathrm{~L}$ and $\mathrm{D} 2 \mathrm{~L}$ 
Fig. 6 Sagital plane of the left knee (medial femoral condyle) from B group in a T2-weighted turbine spin echo fat saturated sequence which demonstrates knee joint effusion

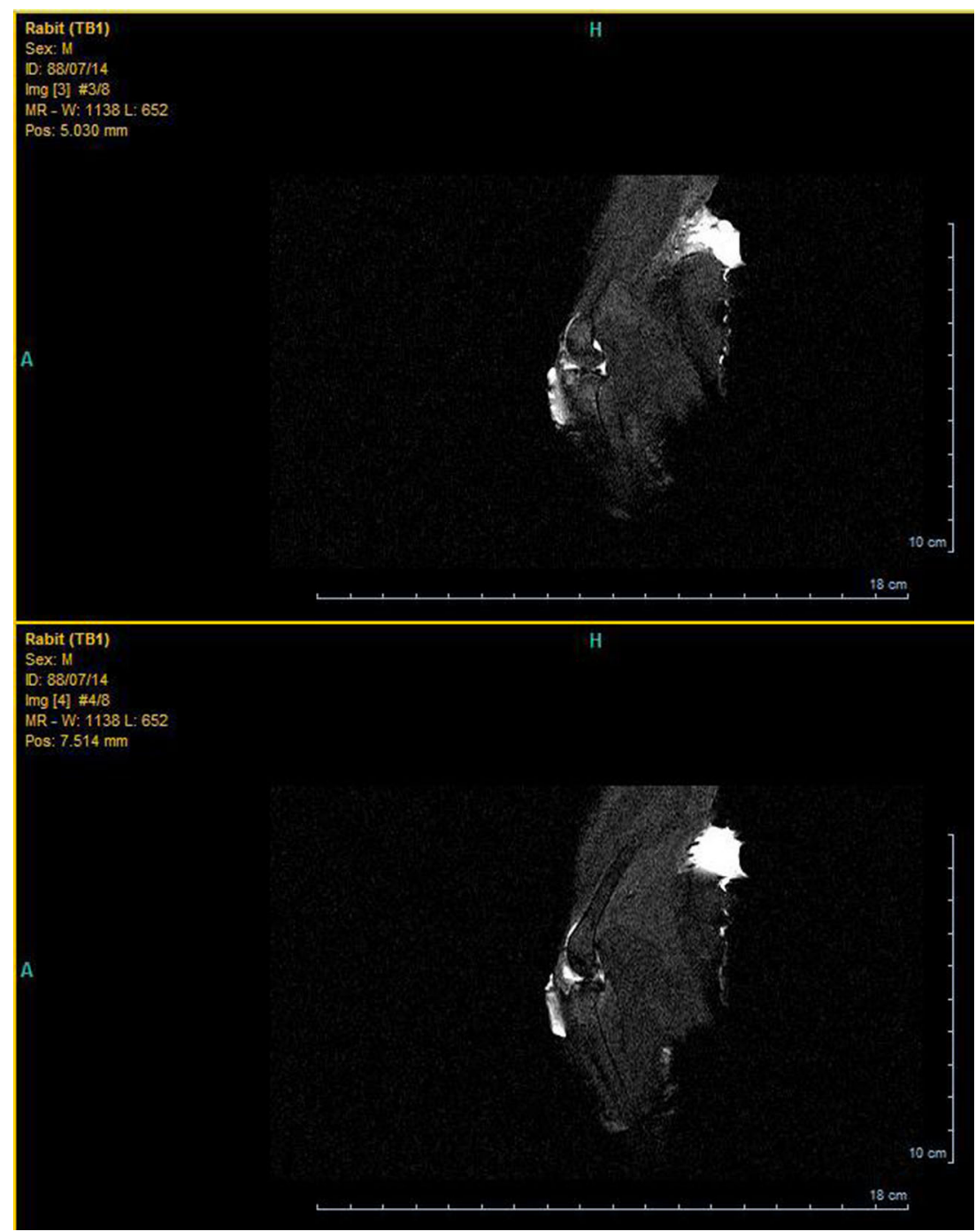

